\title{
Spectral properties of grain boundaries at small angles of rotation
}

\author{
Rainer Hempel and Martin Kohlmann
}

\begin{abstract}
We study some spectral properties of a simple two-dimensional model for small angle defects in crystals and alloys. Starting from a periodic potential $V: \mathbb{R}^{2} \rightarrow \mathbb{R}$, we let $V_{\vartheta}(x, y)=V(x, y)$ in the right half-plane $\{x \geq 0\}$ and $V_{\vartheta}=V \circ M_{-\vartheta}$ in the left half-plane $\{x<0\}$, where $M_{\vartheta} \in \mathbb{R}^{2 \times 2}$ is the usual matrix describing rotation of the coordinates in $\mathbb{R}^{2}$ by an angle $\vartheta$. As a main result, it is shown that spectral gaps of the periodic Schrödinger operator $H=-\Delta+V$ fill with spectrum of $R_{\vartheta}=-\Delta+V_{\vartheta}$ as $0 \neq \vartheta \rightarrow 0$. Moreover, we obtain upper and lower bounds for a quantity pertaining to an integrated density of states measure for the surface states.
\end{abstract}

Mathematics Subject Classification (2010). Primary 35J10; Secondary 35P20, 81Q10.

Keywords. Schrödinger operators, eigenvalues, spectral gaps.

\section{Introduction}

In the quantum theory of solids one first studies periodic structures which can often be modelled by Schrödinger operators with periodic potentials. Other models deal with situations where periodicity holds only in subsets of the sample; more precisely, the sample is the disjoint union of subsets such that, in each subset, the potential is obtained by restricting different periodic potentials to the corresponding subsets. Such zones or "grains" occur frequently in crystals and in alloys; some typical examples are shown in Figure 1. It is an important issue to understand how the interface between two grains will influence the energy spectrum of the sample. Typically, the grain boundaries appear to be (piecewise) linear, and one is led to study problems on $\mathbb{R}^{2}$ with a potential $W=W(x, y)$ defined by

$$
W(x, y)= \begin{cases}V_{r}(x, y) & x \geq 0, \\ V_{\ell}(x, y) & x<0,\end{cases}
$$

where $V_{r}, V_{\ell}: \mathbb{R}^{2} \rightarrow \mathbb{R}$ are periodic. In many situations, $V_{\ell}$ is obtained from $V_{r}$ by a translation or a rotation about the origin. 

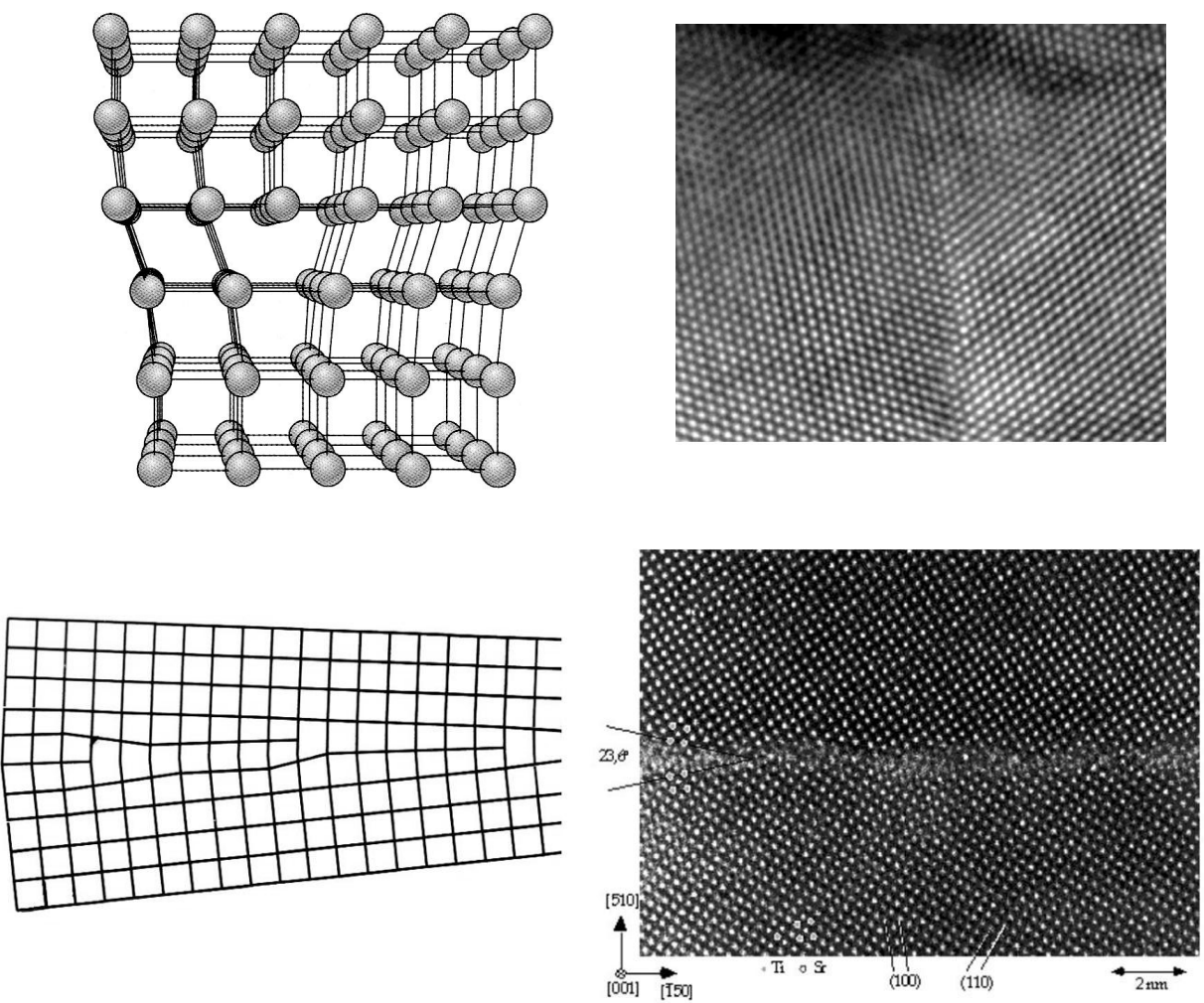

Figure 1. Edge dislocation and small angle grain boundary. The pictures on the left are from [15] with kind permission of the publisher. The pictures on the right were taken with a TEM. References

- $\quad$ http://www.fys.uio.no/bate/?page_id=7

- http://pruffle.mit.edu/ ccarter/NANOAM/images/

In this paper, we will use some results on a translational problem to obtain spectral information about rotational problems in the limit of small angles. Our main theorem deals with the following situation. Let $V: \mathbb{R}^{2} \rightarrow \mathbb{R}$ be a Lipschitz-continuous function which is periodic w.r.t. the lattice $\mathbb{Z}^{2}$. For $\vartheta \in(0, \pi / 2)$; let

$$
M_{\vartheta}=\left(\begin{array}{cc}
\cos \vartheta & -\sin \vartheta \\
\sin \vartheta & \cos \vartheta
\end{array}\right) \in \mathbb{R}^{2 \times 2},
$$

and

$$
V_{\vartheta}(x, y)= \begin{cases}V(x, y) & x \geq 0 \\ V\left(M_{-\vartheta}(x, y)\right) & x<0 .\end{cases}
$$

We then let $H_{0}$ denote the (unique) self-adjoint extension of $-\Delta \uparrow C_{c}^{\infty}\left(\mathbb{R}^{2}\right)$, acting 
in the Hilbert space $L_{2}\left(\mathbb{R}^{2}\right)$, and

$$
R_{\vartheta}=H_{0}+V_{\vartheta}, \quad D\left(R_{\vartheta}\right)=D\left(H_{0}\right) .
$$

Then $R_{\vartheta}$ is essentially self-adjoint on $C_{c}^{\infty}\left(\mathbb{R}^{2}\right)$ and semi-bounded from below. Our main assumption is that the periodic Hamiltonian $H=H_{0}+V=R_{0}$ has a gap $(a, b)$ in the essential spectrum $\sigma_{\text {ess }}(H)$, i.e., we assume that there exist $a<b \in \mathbb{R}$ that satisfy inf $\sigma_{\text {ess }}(H)<a$ and $(a, b) \cap \sigma(H)=\emptyset$; we do not need to assume that $a$, $b$ are the actual gap edges. It is easy to see (using, e.g., Theorem VIII.25 in [20]) that the operators $R_{\vartheta}$ converge to $R_{\vartheta_{0}}$ in the strong resolvent sense as $\vartheta \rightarrow \vartheta_{0} \in[0, \pi / 2)$; in particular, $R_{\vartheta}$ converges to $H$ in the strong resolvent sense as $\vartheta \rightarrow 0$. Recall that strong resolvent convergence implies upper semi-continuity of the spectrum while the spectrum may contract considerably when the limit is reached. In the present paper, we are dealing with a situation where the spectrum in fact behaves discontinuously at $\vartheta=0$ since, counter to first intuition, the spectrum of $R_{\vartheta}$ "fills" the gap $(a, b)$ as $\vartheta \rightarrow 0$ with $\vartheta>0$. This implies, in particular, that $R_{\vartheta}$ cannot converge to $H$ in the norm resolvent sense, as $\vartheta \rightarrow 0$.

Theorem 1.1. Let $H, R_{\vartheta}$ and $(a, b)$ as above. Then, for any $\varepsilon>0$ there exists $0<\vartheta_{\varepsilon}<\pi / 2$ such that for any $E \in(a, b)$ we have

$$
\sigma\left(R_{\vartheta}\right) \cap(E-\varepsilon, E+\varepsilon) \neq \emptyset, \quad \forall 0<\vartheta<\vartheta_{\varepsilon} .
$$

Remarks 1.2. (i) Roughly speaking, the moment we start rotating the potential on the left-hand side by a tiny angle the gap $(a, b)$ is suddenly full of spectrum of $R_{\vartheta}$ in the sense that, for $0<\vartheta<\vartheta_{\varepsilon}$, no gap of $R_{\vartheta}$ in the interval $(a, b)$ can have length larger than $2 \varepsilon$. It is conceivable that for most $\vartheta$ the spectrum of $R_{\vartheta}$ covers the interval $(a, b)$, but there are examples (cf. Section 5) where $R_{\vartheta}$ has gaps in $(a, b)$ for some $\vartheta$.

(ii) It seems to be quite hard to determine the nature of the spectrum of $R_{\vartheta}$ for general $\vartheta \in(0, \pi / 2)$; however, there are some special angles for which a result from [6] excludes singular continuous spectrum (cf. Section 6).

(iii) In addition to what is stated in Theorem 1.1 we obtain lower and upper bounds for the spectral densities in the intervals $(E-\varepsilon, E+\varepsilon)$ on a scale that is appropriate to surface states (without knowing that an integrated surface density of states as in [9] and [18] exists for $R_{\vartheta}$ ); cf. Theorems 4.1 and 4.3.

There is a simple, intuitive connection between the rotational problem and the related translational problem, given as follows. Starting from the same periodic potential $V$ as above, we now look at

$$
W_{t}(x, y)=\left\{\begin{array}{ll}
V(x, y) & x \geq 0, \\
V(x+t, y) & x<0,
\end{array} \quad 0 \leq 1 \leq t,\right.
$$

and define $D_{t}=-\Delta+W_{t}$, acting in $L_{2}\left(\mathbb{R}^{2}\right)$. In the 1-dimensional case, this problem has been studied in great detail by Korotyaev in [16] and [17]. A different approach 
was most recently implemented in [12] where it is shown that some of the results of [16] and [17] can be recovered with a rather crude variational technique. This method can be easily generalized to the dislocation problem on a strip $\mathbb{R} \times(0,1)$ and then to the plane $\mathbb{R}^{2}$; one finds that spectrum of $D_{t}$ crosses the gap as $t$ varies between 0 and 1. Now our key observation consists in the following: for any given $\varepsilon>0$ and $n \in \mathbb{N}$, we can find points $(0, \eta)$ on the $y$-axis such that

$$
\left|V_{\vartheta}(x, y)-W_{t}(x, y)\right|<\varepsilon, \quad(x, y) \in Q_{n}(0, \eta),
$$

with $Q_{n}(0, \eta)=(-n, n) \times(\eta-n, \eta+n)$, provided $\vartheta>0$ is small enough and satisfies a condition which ensures an appropriate alignment of the period cells on the $y$-axis. This basic observation is somewhat reminiscent of a key idea in [11] where rotationally symmetric Schrödinger operators of the type $-\Delta+U(|x|)$ in $L_{2}\left(\mathbb{R}^{n}\right)$ with $U: \mathbb{R} \rightarrow \mathbb{R}$ periodic and Lipschitz-continuous are considered: here, far away from the origin, the potential $U(|x|)$ looks very much like a potential depending only on the $x_{1}$-coordinate if we restrict our attention to balls of fixed size with centers far out on the $x_{1}$-axis.

The paper is organized as follows. In Section 2, we briefly summarize some results of [12] on translational lattice dislocations for the strip and for the plane. What we will use in the sequel is the simple fact that, for any $E \in(a, b)$, there is some $t=t_{E} \in(0,1)$ with the following property: for any $\varepsilon>0$, there is a compactly supported approximate eigenfunction $u$ in the domain of $D_{t}$ that satisfies $\left\|\left(D_{t}-E\right) u\right\|<\varepsilon$ and $\|u\|=1$.

In Section 3, we first employ the Birkhoff ergodic theorem to obtain a set $\Theta \subset$ $(0, \pi / 2)$ with countable complement so that (3) can be established for small $\vartheta \in \Theta$ and suitable $\eta \in \mathbb{R}$. Then the above approximate eigenfunctions $u$ will also be approximate eigenfunctions of $R_{\vartheta}$ for small $\vartheta \in \Theta$, after an appropriate ( $\vartheta$-dependent) translation along the $y$-axis. This then gives Theorem 1.1 .

Suitable points $(0, \eta)$ for the construction of Section 3 occur with a certain density and we expect a lower bound for the integrated surface density of states. Since we do not know whether the i.d.s. measure or the integrated surface density of states measure exist, we only provide lower and upper bounds for the number of Dirichlet eigenvalues in subintervals of the gap $(a, b)$ for our operators $R_{\vartheta}$, restricted to large squares $Q_{n}=(-n, n)^{2}$. Theorem 4.1 in Section 4 provides a lower bound of the form $c_{1} n$ for $n$ large with a positive constant $c_{1}$, while Theorem 4.3 gives an upper bound by $c_{2} n \log n$, for $n$ large. Note that Theorem 4.3 deals with a much more general situation: in fact, we allow here for two different potentials $V_{\ell}$ and $V_{r}$ on the left and right which are not required to be periodic; all we need is a common gap.

In Section 5 we discuss examples of "muffin tin"-type which come in three versions: in the simplest case, the muffin tins are circular wells, arranged on a periodic grid, with infinitely high walls (so that the Schrödinger operator is just the direct sum of a countable number of Dirichlet Laplacians on circles). We then approximate by muffin tins of finite height, and, finally, by muffin tins with Lipschitz potentials. We obtain spectral results for the rotation problem for all three versions. 
In Section 6, finally, we first explain a simplified model for small angle grain boundaries where we assume rotation by an angle $\vartheta / 2$ in both half-planes in opposite direction; this problem is somewhat easier to deal with. We furthermore discuss special rotation angles $\vartheta$ for which the operators $R_{\vartheta}$ are periodic in the $y$-direction and thus have no singular continuous spectrum [6]. All these question hinge on an approximate or exact matching up between the given periodic potential in the right half-plane and its rotated version in the left half-plane. This is somewhat connected with the question of coincidence between a lattice and some of its rotated versions (CSL-lattices).

For basic results on the spectral theory of self-adjoint operators or, more specifically, periodic Schrödinger operators, we refer to [14], [21], [8], and [19]. We will use results from these sources mostly without specific reference.

The authors would like to thank Andreas Ruschhaupt, University of Hannover, for several fruitful discussions and the unknown referee for suggestions that helped to improve the presentation.

\section{The dislocation problem on a strip and for the plane}

Let $V: \mathbb{R}^{2} \rightarrow \mathbb{R}$ be $\mathbb{Z}^{2}$-periodic and Lipschitz-continuous, let $I=(0,1)$, and let $\Sigma=\mathbb{R} \times(0,1)=\mathbb{R} \times I$ denote the infinite strip of width 1 . As above, we write $H=-\Delta+V$ for the (self-adjoint) Schrödinger operator with potential $V$ acting in $L_{2}\left(\mathbb{R}^{2}\right)$. Then $\sigma(H)$, the spectrum of $H$, has band structure, i.e. it is the (locally finite) union of compact intervals [21]. The intervals of spectrum, the bands, may be separated by (open) intervals, the gaps. Moreover, $\sigma(H)$ is purely absolutely continuous. For $0 \leq t \leq 1$, we introduce the self-adjoint operators

$$
\begin{array}{cl}
S_{t}=-\Delta+W_{t} & \text { acting in } L_{2}(\Sigma), \\
D_{t}=-\Delta+W_{t} & \text { acting in } L_{2}\left(\mathbb{R}^{2}\right),
\end{array}
$$

where $S_{t}$ has periodic boundary conditions in the $y$-variable and $W_{t}$ is as in (2). Since $V$ is bounded, the domains $D(\cdot)$ of the above operators satisfy $D\left(D_{t}\right)=D(H)$ and $D\left(S_{t}\right)=D\left(H_{0, \Sigma}\right)$ for all $t$, where $H_{0, \Sigma}$ denotes the Laplacian on $\Sigma$ with periodic boundary conditions in $y$. The operator $-\Delta+W_{t}$ in $L_{2}(\Sigma)$ with $\vartheta$-periodic boundary conditions in $y$ is denoted by $S_{t}(\vartheta)$ for $0 \leq \vartheta \leq 2 \pi$. As usual, $D_{t}$ can be obtained from the $S_{t}(\vartheta)$ as a direct fiber integral,

$$
D_{t}=\int_{0 \leq \vartheta \leq 2 \pi}^{\oplus} S_{t}(\vartheta) \frac{d \vartheta}{2 \pi}
$$

direct fiber integrals are discussed, e.g., in [21] and [6]. As a consequence, for any $\vartheta$ the spectrum of $S_{t}(\vartheta)$ is a subset of $\sigma\left(D_{t}\right)$. Furthermore, using the periodicity in the $x$-direction, each $S_{t}(\vartheta)$ can itself be written as a direct fiber integral and so the spectrum of $S_{t}(\vartheta)$ is purely essential spectrum with a band-gap structure. 
We now give a condensed account of some of the results in [12] concerning the operators $S_{t}$ and $D_{t}$. We begin with $S_{t}$ where we first note the following well-known basic facts.

(1) Adding in a Dirichlet boundary condition on any given vertical line segment $\{(x, y) \in \Sigma: x=\xi\}$, for some $\xi \in \mathbb{R}$, leads to a compact perturbation of the resolvent of $S_{t}$, for all $t$. In fact, if $H_{0, \Sigma, D}$ is defined as $H_{0, \Sigma}$ above, but now with an additional Dirichlet boundary condition at $x=\xi$, one can write down explicit formulae for the integral kernels of the resolvents by using the reflection principle. One finds that the operator $\left(H_{0, \Sigma}+1\right)^{-1}-\left(H_{0, \Sigma, D}+1\right)^{-1}$ is Hilbert-Schmidt. It is then easy to show that $\left(H_{0, \Sigma}+W_{t}+c\right)^{-1}-\left(H_{0, \Sigma, D}+W_{t}+c\right)^{-1}$ is compact for all sufficiently large $c \geq 0$.

(2) It follows from (1) that $\sigma_{\text {ess }}\left(S_{t}\right)=\sigma_{\text {ess }}\left(S_{0}\right)$. To see this, just add in Dirichlet boundary conditions at $x=0$ into $S_{0}$ and $S_{t}$, and another Dirichlet boundary condition at $x=-t$ into $S_{t}$ to obtain the operators $S_{0, D}$ and $S_{t, D}$. Then the parts of $S_{0, D}$ and $S_{t, D}$ on $(0, \infty) \times I$ are equal while the contribution to $S_{t, D}$ from $(-\infty,-t) \times I$ is unitarily equivalent to the part of $S_{0, D}$ in $(-\infty, 0) \times I$. Finally, the part of $S_{t, D}$ associated with $(-t, 0) \times I$ has compact resolvent.

(3) The eigenvalues of $S_{t}$ in the gaps of $S_{0}$ are continuous functions of the parameter $t$. (A simple proof can be obtained by scaling the interval $(-n-t, n)$ in such a way that we can use $L_{2}((-n, n) \times I)$ as a common Hilbert space).

The following result shows that there is non-trivial spectral flow of the family $S_{t}$ through the gaps of $S_{0}$.

Proposition 2.1. Let $(a, b)$ denote a spectral gap of $H$ and let $E \in(a, b)$. Then there exists some $t=t_{E} \in(0,1)$ such that $E$ is a (discrete) eigenvalue of $S_{t_{E}}$.

Moreover, for any $n \in \mathbb{N}$ there are functions $v_{n}=v_{n}(x, y)$ in the domain of $S_{t}$ that satisfy $\left\|v_{n}\right\|=1$, supp $v_{n} \subset[-n, n] \times[0,1]$ and $\left(S_{t_{E}}-E\right) v_{n} \rightarrow 0$ as $n \rightarrow \infty$.

For the proof of Proposition 2.1, we use an approximation by operators with compact resolvent on finite sections of $\Sigma$ (the basic idea is somewhat reminiscent of [7] and [2]): For $n \in \mathbb{N}$, let $S_{t}^{(n)}$ denote the operators $-\Delta+W_{t}$ on $\Sigma_{t}^{(n)}=(-n-$ $t, n) \times I$ with periodic boundary conditions in both variables. The spectrum of the operators $S_{t}^{(n)}$ is purely discrete. Again, the eigenvalues of $S_{t}^{(n)}$ depend continuously on $t$. Furthermore, routine arguments from Floquet theory imply that

(i) $\sigma\left(S_{0}^{(n)}\right) \subset \sigma\left(S_{0}\right)$, for all $n \in \mathbb{N}$, and

(ii) for any gap $(a, b)$ of $S_{0}$ there exists a fixed number $m \in \mathbb{N}$ such that the operators $S_{0}^{(n)}$ (respectively, $S_{1}^{(n)}$ ) have precisely $2 n m$ (respectively, $(2 n+1) m$ ) eigenvalues below $a$, counting multiplicities (cf., e.g., the proof of Theorem XIII.101 in [21] or Theorem 6.2.1 in [8]).

As $t$ increases from 0 to 1 , the operators $S_{0}^{(n)}$ are transformed into $S_{1}^{(n)}$. It is now immediate from property (ii) and the continuity of the eigenvalues that, as $t$ grows from 0 to 1 , at least one eigenvalue of $S_{t}^{(n)}$ must cross $E$. 
We have thus shown that, for each $n \in \mathbb{N}$, there is some $t_{n} \in(0,1)$ and an eigenfunction $u_{n} \in D\left(S_{t_{n}}^{(n)}\right)$ such that $\left\|u_{n}\right\|=1$ and $S_{t_{n}}^{(n)} u_{n}=E u_{n}$. Since the $u_{n}$ obey periodic boundary conditions with respect to the $x$-variable on $\Sigma_{t_{n}}^{(n)}=$ $\left(-n-t_{n}, n\right) \times I$, we can use routine arguments to show that the parts of $u_{n}$ (and of $\left.\nabla u_{n}\right)$ outside of $(-n / 2, n / 2)$ go to zero, as $n \rightarrow \infty$. Indeed, let us fix cut-off functions $\varphi_{n}=\varphi_{n}(x) \in C_{c}^{\infty}(-n / 2, n / 2)$ that satisfy $\varphi_{n}(x)=\varphi_{1}(x / n)$ and $\varphi_{1}(x)=1$ for $-1 / 4 \leq x \leq 1 / 4$. Then $r_{n}=\left(1-\varphi_{n}\right) u_{n}$ vanishes in $(-n / 4, n / 4) \times I$. We now define $\tilde{r}_{n}$ by $\tilde{r}_{n}(x, y)=r_{n}(x, y)$, for $0<x<n$, and $\tilde{r}_{n}(x, y)=r_{n}\left(x-2 n-t_{n}, y\right)$, for $x>n$, i.e. we translate the part of $r_{n}$ in $\left(-n-t_{n}, 0\right) \times I$ to the right by $2 n+t_{n}$. Since $r_{n}$ satisfies periodic boundary conditions, it is clear that $\tilde{r}_{n}$ belongs to the domain of the periodic operator $S_{0}$ and that $\left(S_{0}-E\right) \tilde{r}_{n} \rightarrow 0$, as $n \rightarrow \infty$. As $E$ is in a gap of $S_{0}$, this implies $\tilde{r}_{n} \rightarrow 0$ and then also $r_{n} \rightarrow 0$. We therefore see that $v_{n}=\varphi_{n} u_{n}$ satisfies

$$
\left\|v_{n}\right\| \rightarrow 1, \quad\left(S_{t_{n}}^{(n)}-E\right) v_{n} \rightarrow 0
$$

as $n \rightarrow \infty$. Without loss of generality we may assume that the $t_{n}$ converge to some $t=t_{E} \in(0,1)$. But $v_{n} \in D\left(S_{t_{n}}\right)$ and $S_{t_{n}} v_{n}=S_{t_{n}}^{(n)} v_{n}$, and we find that $\left(S_{t_{E}}-E\right) v_{n} \rightarrow 0$, as $n \rightarrow \infty$ (recall that $V$ is Lipschitz-continuous). Now the spectral theorem implies $E \in \sigma\left(S_{t_{E}}\right)$, as required. Furthermore, the functions $v_{n}$ clearly enjoy the property stated in the second part of Proposition 2.1.

The functions $v_{n}$ constructed above satisfy periodic boundary conditions with respect to $y$ and may thus be extended to $y$-periodic functions $\tilde{v}_{n}$ on $\mathbb{R}^{2}$. Applying also cut-offs $\psi_{n}=\varphi_{n}(y)$ in the $y$-direction, we let

$$
w_{n}=\frac{1}{\left\|\psi_{n} \tilde{v}_{n}\right\|} \psi_{n} \tilde{v}_{n}
$$

the $w_{n}$ satisfy $\left\|w_{n}\right\|=1, w_{n} \in D_{t_{n}}$ and $\left(D_{t_{n}}-E\right) w_{n} \rightarrow 0$ as $n \rightarrow \infty$. By the same argument as above this leads to $E \in \sigma\left(D_{t_{E}}\right)$ (where, again $t_{E}=\lim t_{n}$ ) and we have thus obtained the following result.

Proposition 2.2. Let $(a, b)$ denote a spectral gap of $H$ and let $E \in(a, b)$. Then there exists $t=t_{E} \in(0,1)$ such that $E \in \sigma\left(D_{t_{E}}\right)$.

Moreover, for any $n \in \mathbb{N}$ there are functions $w_{n}=w_{n}(x, y)$ in the domain of $D_{t}$ that satisfy $\left\|w_{n}\right\|=1$, supp $w_{n} \subset[-n, n]^{2}$ and $\left(D_{t_{E}}-E\right) w_{n} \rightarrow 0$ as $n \rightarrow \infty$.

Note that the spectrum of $D_{t}$ inside $(a, b)$ will again consist of bands which we could find by repeating the above process for all $\vartheta$-periodic boundary conditions w.r.t. $y$. For a detailed discussion and further results, we refer to [12].

\section{The rotation problem for small angles}

In this section, we study the spectrum of the operators $R_{\vartheta}$, for $0<\vartheta<\pi / 2$, where the $R_{\vartheta}$ are defined in (1) as self-adjoint operators in the Hilbert space $L_{2}\left(\mathbb{R}^{2}\right)$. 
In view of a proof of Theorem 1.1, consider a fixed $E \in(a, b)$. Then, by Proposition 2.1, there is some $t \in(0,1)$ such that $E$ is in the spectrum of the dislocation operator $D_{t}$ on the plane. We wish to find angles $\vartheta$ with the property that the potential $V_{\vartheta}$ is approximately equal to $W_{t}$ on a sufficiently large square $Q_{n}(0, \eta)$ of side-length $2 n$, centered at some point $(0, \eta)$ on the $y$-axis. This leads to the following requirements: If we imagine the grid $\Gamma=\left\{(x, y) \in \mathbb{R}^{2}: x \in \mathbb{Z}\right.$ or $\left.y \in \mathbb{Z}\right\}$ of lines describing the period cells, we have to make sure that, inside $Q_{n}(0, \eta)$, the alignment between the horizontal lines of $\Gamma$ in the right half-plane with the rotated horizontal lines of $M_{\vartheta} \Gamma$ in the left half-plane is nearly perfect on the $y$-axis and that the rotated vertical lines of $M_{\vartheta} \Gamma$ in the left half-plane have, roughly, distance $t$ (modulo $\mathbb{Z}$ ) from the $y$-axis. More precisely, we wish to find $m \in \mathbb{N}$ such that $m / \cos \vartheta$ is integer, up to a small error, and $m \tan \vartheta=t(\bmod \mathbb{Z})$, again up to a small error, inside $Q_{n}(0, \eta)$.

We first prepare a lemma which deals with ergodicity on the flat torus $\mathbb{T}^{2}=$ $\mathbb{R}^{2} / \mathbb{Z}^{2}$, as in [20] and [5]. We consider transformations $T_{\vartheta}: \mathbb{T}^{2} \rightarrow \mathbb{T}^{2}$ defined by

$$
T_{\vartheta}(x, y)=(x+\tan \vartheta, y+1 / \cos \vartheta) .
$$

Lemma 3.1. There is a set $\Theta \subset(0, \pi / 2)$ with countable complement such that the transformation $T_{\vartheta}$ in (4) is ergodic for all $\vartheta \in \Theta$.

Proof. $T_{\vartheta}$ is ergodic if and only if the numbers $1, \tan \vartheta$, and $1 / \cos \vartheta$ are independent over the rationals, i.e. $\left(n_{1}, n_{2}, n_{3}\right) \in \mathbb{Z}^{3}$ and

$$
n_{1}+n_{2} \tan \vartheta+\frac{n_{3}}{\cos \vartheta}=0
$$

implies $n_{1}=n_{2}=n_{3}=0$. Write $\mathbb{Z}_{3}=\mathbb{Z}^{3} \backslash\{(0,0,0)\}$. For any triple $\left(n_{1}, n_{2}, n_{3}\right) \in \mathbb{Z}_{3}$ the set of points $(x, y) \in \mathbb{R}^{2}$ that satisfy $n_{1}+n_{2} x+n_{3} y=0$ is a line $\ell=\ell_{\left(n_{1}, n_{2}, n_{3}\right)} \subset \mathbb{R}^{2}$. Consider the (countable) set

$$
\Lambda=\left\{\ell_{\left(n_{1}, n_{2}, n_{3}\right)}:\left(n_{1}, n_{2}, n_{3}\right) \in \mathbb{Z}_{3}\right\} .
$$

In (5), the variables $x$ and $y$ are of the special form $x=\tan \vartheta, y=1 / \cos \vartheta$ and so $y=\sqrt{1+x^{2}}$. Since $F(x)=\sqrt{1+x^{2}}$ is convex, each $\ell \in \Lambda$ has at most two intersection points with the graph $G(F)$ of $F$. Then

$$
S=\bigcup_{\ell \in \Lambda}\{\ell \cap G(F)\}
$$

is countable and so $G(F) \backslash S$ has full 1-dimensional measure. Let $G(F)_{+}=G(F) \cap$ $\{x>0\}$. We map $G(F)_{+} \backslash S$ to $(0, \pi / 2)$ by $h:(x, F(x)) \mapsto \arctan x$. Since $h: G(F)_{+} \rightarrow(0, \pi / 2)$ is diffeomorphic, $\Theta=h\left(G(F)_{+} \backslash S\right)$ is as desired.

Let us write $x_{\sim}$ for the fractional part of $x>0$, i.e. $x_{\sim}=x-\lfloor x\rfloor$ if $x>0$. In the proof of our main theorem, we will need natural numbers $m$ such that, for 
$t \in(0,1)$ given, $(m \tan \vartheta)_{\sim}$ is approximately equal to $t$ and $(m / \cos \vartheta)_{\sim}$ almost equals 0 . The existence of such numbers $m$ follows from Lemma 3.1 and Birkhoff's ergodic theorem. Let $\vartheta \in \Theta, \varepsilon>0$, and let us denote by $\chi_{Q}$ the characteristic function of the set $Q=(t-\varepsilon, t+\varepsilon) \times(-\varepsilon, \varepsilon) \subset \mathbb{T}^{2}$. Then, for all $(x, y) \in \mathbb{T}^{2}$,

$$
\lim _{n \rightarrow \infty} \frac{1}{n} \sum_{m=0}^{n-1} \chi_{Q}\left(T_{\vartheta}^{m}(x, y)\right)=\int_{Q} \mathrm{~d} x \mathrm{~d} y=4 \varepsilon^{2}>0,
$$

and we may take $(x, y)=(0,0)$ to arrive at the desired result.

We add the following remarks to the above argument.

(1) Translation on the torus is a particularly simple ergodic transformation: for $\vartheta$ given, it can equivalently be seen as linear motion on parallel lines in $\mathbb{R}^{2}$, factored by $\mathbb{Z}^{2}$. In particular, two nearby points $(x, y)$ and $\left(x^{\prime}, y^{\prime}\right)$ will forever keep their relative position under the action of $T_{\vartheta}^{m}$, and thus the statement of Birkhoff's theorem holds for any point $(x, y)$, not just for a.e. ( $x, y)$ (cf., e.g., Chapter $3, \S 1$ in [5]).

(2) In some sense, Birkhoff's theorem is the strongest result one can use in this context. Similar results are obtained from Dirichlet's theorem on the approximation of irrational numbers by rationals.

We are now ready for a first main result which establishes the existence of surface states in the gaps of $H$ and shows that, in fact, any gap $(a, b)$ of $H$ is filling up with spectrum of $R_{\vartheta}$ as $\vartheta \rightarrow 0$.

Proposition 3.2. Let $(a, b)$ be a spectral gap of $H$ and let $[\alpha, \beta] \subset(a, b), \alpha<\beta$. Then there is a $\vartheta_{0}=\vartheta_{0}(\alpha, \beta)>0$ such that

$$
\sigma\left(R_{\vartheta}\right) \cap(\alpha, \beta) \neq \varnothing, \quad \forall \vartheta \in\left(0, \vartheta_{0}\right) .
$$

Proof. (1) We first restrict our attention to $\vartheta \in \Theta$ with $\Theta$ as in Lemma 3.1. Let $E \in(\alpha, \beta)$ and $\varepsilon=\min \{E-\alpha, \beta-E\} / 2$. By Proposition 2.1, we can find $n=n_{\varepsilon} \in \mathbb{N}$ and a function $u_{n}$ of norm 1 in the domain of $D_{t}$ with supp $u_{n} \subset[-n, n]^{2}$ such that $\left\|\left(D_{t}-E\right) u_{n}\right\|<\varepsilon$. Obviously $u_{n, k}(x, y)=u_{n}(x, y-k)$ satisfies the same estimate for any $k \in \mathbb{N}$. If we can show that, for appropriate $k \in \mathbb{N}$,

$$
\left|V_{\vartheta}(x, y)-W_{t}(x, y)\right|<\varepsilon, \quad(x, y) \in Q_{n}(0, k)
$$

(recall the definition of $\left.Q_{n}(0, k)=(-n, n) \times(k-n, k+n)\right)$, we may conclude that

$$
\left\|\left(R_{\vartheta}-E\right) u_{n, k}\right\|<2 \varepsilon,
$$

but then the spectral theorem implies that $R_{\vartheta}$ has spectrum inside the interval ( $E-2 \varepsilon$, $E+2 \varepsilon) \subset(\alpha, \beta)$.

For a proof of (7), we first observe that by the properties of $V$ and the definitions of $V_{\vartheta}$ and $W_{t}$, we have the following estimate:

$$
\left|V_{\vartheta}(x, y)-W_{t}(x, y)\right|^{2} \leq \min _{j_{1}, j_{2} \in \mathbb{Z}} L^{2}\left(\left(X-j_{1}\right)^{2}+\left(Y-j_{2}\right)^{2}\right),
$$


for all $(x, y) \in \mathbb{R}^{2}$, with

$$
X=x(\cos \vartheta-1)-t+y \sin \vartheta, \quad Y=-x \sin \vartheta+y(\cos \vartheta-1)
$$

and $L$ the Lipschitz constant of $V$. Now for $\vartheta \in \Theta$ given, there is some $m=m_{\vartheta} \in \mathbb{N}$ such that

$$
\left(\frac{m}{\cos \vartheta}\right)_{\sim}<\varepsilon / 4, \quad\left|(m \tan \vartheta)_{\sim}-t\right|<\varepsilon / 4
$$

in particular, there is some $N \in \mathbb{N}$ such that $|m / \cos \vartheta-N|<\varepsilon / 4$.

We may now apply the estimate (8) to the points $(x, y) \in Q_{n}(0, N)$ to find

$$
\left|V_{\vartheta}(x, y)-W_{t}(x, y)\right|^{2} \leq L^{2}\left((X-\lfloor m \tan \vartheta\rfloor)^{2}+(Y+N-m)^{2}\right),
$$

for all $(x, y) \in Q_{n}(0, N)$. Here

$$
\begin{aligned}
|X-\lfloor m \tan \vartheta\rfloor| & \leq n(1-\cos \vartheta)+n \vartheta+|m \tan \vartheta-\lfloor m \tan \vartheta\rfloor-t| \\
& \leq 2 n_{\varepsilon} \vartheta+|(m \tan \vartheta) \sim-t|
\end{aligned}
$$

and

$$
|Y+N-m| \leq 2 n_{\varepsilon} \vartheta+|N-m / \cos \vartheta|
$$

We choose $\vartheta_{0}>0$ small enough to have $2 n_{\varepsilon} \vartheta_{0}<\varepsilon / 4$ and (7) follows if we pick $k=N$. We have thus shown that $R_{\vartheta}$ has spectrum in $(\alpha, \beta)$ for all $\vartheta \in \Theta \cap\left(0, \vartheta_{0}\right)$.

(2) In order to remove the restriction $\vartheta \in \Theta$ we note that with each $\vartheta \in \Theta$ there comes a positive number $\eta_{\vartheta}>0$ such that

$$
\left\|\left(R_{\sigma}-E\right) u_{n, k}\right\|<3 \varepsilon, \quad \forall \sigma \in\left(\vartheta-\eta_{\vartheta}, \vartheta+\eta_{\vartheta}\right)
$$

since

$$
\left\|\left(V_{\sigma}-V_{\vartheta}\right) \uparrow \operatorname{supp} u_{n, k}\right\|_{\infty} \rightarrow 0, \quad \sigma \rightarrow \vartheta .
$$

As the intervals $\left(\vartheta-\eta_{\vartheta}, \vartheta+\eta_{\vartheta}\right)$ with $\vartheta$ ranging between 0 and $\vartheta_{0}$ cover the interval $\left(0, \vartheta_{0}\right)$, the desired result follows.

Now it is easy to obtain Theorem 1.1 in the Introduction from Proposition 3.2.

Proof of Theorem 1.1. For $\varepsilon>0$ given, we consider points $a=\gamma_{0}<\gamma_{1}<\gamma_{2}<$ $\cdots<\gamma_{N}=b$ such that $\gamma_{j}-\gamma_{j-1}<\varepsilon / 2$, for $j=1, \ldots, N$. For each of the intervals $I_{j}=\left(\gamma_{j-1}, \gamma_{j}\right), 2 \leq j \leq N-1$, Proposition 3.2 yields a constant $\vartheta_{j}>0$ with the property that $R_{\vartheta}$ has spectrum in the interval $I_{j}$ for all $0<\vartheta<\vartheta_{j}$. Then $\vartheta_{0}=\min _{2 \leq j \leq N-1} \vartheta_{j}$ has the required properties. 


\section{Integrated density of states bounds}

It is clear that ergodicity gives us not just a single $m$ as in (9), for $\vartheta \in \Theta$; in fact, (6) guarantees that suitable $m$ will appear with a certain frequency. We will use this observation to obtain lower bounds for a quantity which, in the limit, would translate into a (positive) lower bound for the surface i.d.s. measure if we knew that the required limit exists. This will be complemented by a similar upper bound which is of the expected order, up to a logarithmic factor. A detailed and rather complete account of the i.d.s. for (random) Schrödinger operators can be found in [22] which also contains a wealth of references. [9] and [18] specifically discuss the existence of a surface i.d.s. as a distribution or a measure. Some results on the surface i.d.s. measure for the translational dislocation problem can be found in [12].

Let $R_{\vartheta}^{(n)}$ denote the operator $-\Delta+V_{\vartheta}$, acting in $L_{2}\left(Q_{n}\right)$ with Dirichlet boundary conditions, where $Q_{n}=(-n, n)^{2} \subset \mathbb{R}^{2}$. For any interval $I \subset \mathbb{R}$, we denote by $N_{I}\left(R_{\vartheta}^{(n)}\right)$ the number of eigenvalues of $R_{\vartheta}^{(n)}$ in $I$, each eigenvalue being counted according to its multiplicity. The existence of a surface i.d.s. measure in the gap $(a, b)$ would correspond to the existence of a finite $\operatorname{limit}_{\lim } \operatorname{li\infty }_{n \rightarrow \infty} N_{I}\left(R_{\vartheta}^{(n)}\right) / n$, for any interval $I$ with $\bar{I} \subset(a, b)$. Theorem 4.1 provides lower bounds of the form

$$
\liminf _{n \rightarrow \infty} \frac{1}{n} N_{I}\left(R_{\vartheta}^{(n)}\right)>0,
$$

for (non-degenerate) subintervals $I$ and small $\vartheta \in \Theta$, while Theorem 4.3 yields an upper bound

$$
\limsup _{n \rightarrow \infty} \frac{1}{n \log n} N_{I}\left(R_{\vartheta}^{(n)}\right)<\infty .
$$

We begin with a lower bound.

Theorem 4.1. Let $H, R_{\vartheta}$ as above and suppose that $(a, b)$ is a spectral gap of $H$. Let $\Theta$ as in Lemma 3.1.

Then, for any $\varepsilon>0$ there exists $a \vartheta_{\varepsilon}>0$ such that (10) holds for all $\vartheta \in$ $\Theta \cap\left(0, \vartheta_{\varepsilon}\right)$ and for any interval $I \subset(a, b)$ of length greater than $\varepsilon$.

Proof. (1) Let $[\alpha, \beta] \subset(a, b)$, fix $E \in(\alpha, \beta)$, and let $0<\varepsilon<\min \{E-\alpha, \beta-E\}$. Let $u_{0}$ in the domain of $D_{t}$ with compact support satisfy $\left\|u_{0}\right\|=1$ and $\left\|\left(D_{t}-E\right) u_{0}\right\|<$ $\varepsilon$, as in Proposition 2.1. Let $v \in \mathbb{N}$ be such that supp $u_{0} \subset Q_{v}=(-v, v)^{2}$; note that, in this proof, $v$ corresponds to the parameter $n$ of Section 3 .

Let $\vartheta \in \Theta \cap(0, \pi / 4]$ so that, in particular, $1 / \sqrt{2} \leq \cos \vartheta \leq 1$. By ergodicity, there exists a constant $c_{0}=c_{0}(\vartheta)>0$ with the following properties: for $n \in \mathbb{N}$ large, there are at least $J_{n}=\left\lfloor c_{0} n\right\rfloor$ natural numbers $m_{1}, \ldots, m_{J_{n}} \in(0, n / 4)$ such that (9) holds for $m=m_{s}, s=1, \ldots, J_{n}$, and such that

$$
\left|m_{s}-m_{r}\right| \geq 2 v, \quad s \neq r, 1 \leq s, r \leq J_{n} ;
$$


here $J_{n}$ and $m_{1}, \ldots, m_{J_{n}}$ depend on $n$ and $\vartheta$. It follows that for each $j=1, \ldots, J_{n}$ there is some $N_{j} \in \mathbb{N}$ such that $\left|m_{s} / \cos \vartheta-N_{j}\right|<\varepsilon / 4$ and $\left|m_{s} \tan \vartheta-t\right| \sim<\varepsilon / 4$. We then see that the functions $\varphi_{j}$, defined by $\varphi_{j}(x, y)=u_{0}\left(x, y-N_{j}\right)$, are of norm 1 and have mutually disjoint supports contained in $(-n, n)^{2}$. Furthermore, for $\vartheta$ small enough, $0<\vartheta<\vartheta_{\varepsilon}$, say, we can show (as in the proof of Proposition 3.2) that an estimate (7) holds on each square $(-v, v) \times\left(N_{j}-v, N_{j}+v\right)$. Thus (7) holds on the support of each $\varphi_{j}$ and it follows that

$$
\left\|\left(R_{\vartheta}^{(n)}-E\right) \varphi_{j}\right\|<\varepsilon, \quad 0<\vartheta<\vartheta_{\varepsilon}, j=1, \ldots, J_{n} .
$$

Then $\mathcal{M}=\operatorname{span}\left\{\varphi_{j}\right\}_{j=1, \ldots, J_{n}}$ has dimension $J_{n}$. Let $\mathcal{N}$ denote the range of the spectral projection $P_{(\alpha, \beta)}\left(R_{\vartheta}^{(n)}\right)$ of $R_{\vartheta}^{(n)}$ associated with the interval $(\alpha, \beta)$ and assume for a contradiction that $\operatorname{dim} \mathcal{N}<J_{n}$. Then we can find a function $v \in \mathcal{M} \cap \mathcal{N}^{\perp}$ of norm 1. By the spectral theorem, $\left\|\left(R_{\vartheta}^{(n)}-E\right) v\right\| \geq \varepsilon$. On the other hand, (12) and $v=\sum_{i=1}^{N} a_{i} \varphi_{i}$ implies $\left\|\left(R_{\vartheta}^{(n)}-E\right) v\right\|<\varepsilon$ because the $\varphi_{j}$ have mutually disjoint supports.

We have therefore shown that for any interval $I=[\alpha, \beta]$ there exists some $\vartheta_{0}>0$ such that (10) holds for all $\vartheta \in \Theta \cap\left(0, \vartheta_{0}\right)$.

(2) Now let $\varepsilon>0$. As in the proof of Theorem 1.1, given at the end of Section 3, we may cover the interval $(a, b)$ by a finite number of subintervals of length $\varepsilon$; applying the result of part (1) we then obtain the desired statement.

Remarks 4.2. (a) It appears that the argument used at the end of the proof of Proposition 3.2 to remove the restriction $\vartheta \in \Theta$ does not work in the context of Theorem 4.1.

(b) It follows from the proof of Theorem 4.1 that $\sigma_{\text {ess }}\left(R_{\vartheta}\right) \cap I \neq \varnothing$ for all $\vartheta \in \Theta \cap\left(0, \vartheta_{\varepsilon}\right)$ and for any interval $I \subset(a, b)$ of length greater than $\varepsilon$.

We now complement the lower estimate established in Theorem 4.1 by an upper bound which is of the expected order, up to a logarithmic factor. Note that we treat a situation which is far more general than the rotation or dislocation problems studied so far. In fact, we will allow for different potentials $V_{1}$ on the left and $V_{2}$ on the right which are only linked by the assumption that there is a common spectral gap; neither $V_{1}$ nor $V_{2}$ are required to be periodic. The proof uses technology which is fairly standard and based on exponential decay estimates for resolvents.

Theorem 4.3. Let $V_{1}, V_{2} \in L_{\infty}\left(\mathbb{R}^{2}, \mathbb{R}\right)$ and suppose that the interval $(a, b) \subset \mathbb{R}$ does not intersect the spectra of the self-adjoint operators $H_{k}=-\Delta+V_{k}, k=1,2$, both acting in the Hilbert space $L_{2}\left(\mathbb{R}^{2}\right)$. Let

$$
W=\chi_{\{x<0\}} \cdot V_{1}+\chi_{\{x \geq 0\}} \cdot V_{2}
$$

and define $H=-\Delta+W$, a self-adjoint operator in $L_{2}\left(\mathbb{R}^{2}\right)$. Finally, we let $H^{(n)}$ denote the self-adjoint operator $-\Delta+W$ acting in $L_{2}\left(Q_{n}\right)$ with Dirichlet boundary 
conditions. Then, for any interval $\left[a^{\prime}, b^{\prime}\right] \subset(a, b)$, we have

$$
\limsup _{n \rightarrow \infty} \frac{1}{n \log n} N_{\left[a^{\prime}, b^{\prime}\right]}\left(H^{(n)}\right)<\infty .
$$

Proof. (1) We write $N(n)=N_{\left[a^{\prime}, b^{\prime}\right]}\left(H^{(n)}\right)$ and note that there is a constant $c_{0} \geq 0$ such that

$$
N(n) \leq c_{0} n^{2}, \quad n \in \mathbb{N} ;
$$

this follows by routine min-max arguments as in Section XIII.15 of [21].

(2) Let us consider the (normalized) eigenfunctions $u_{i, n}$ of $H^{(n)}$ associated with the eigenvalues $E_{i, n} \in\left[a^{\prime}, b^{\prime}\right]$, for $i=1, \ldots, N(n)$. The main idea of the proof is to show that the $u_{i, n}$ are concentrated near the boundary of $Q_{n}$ or near the $y$-axis. To obtain the corresponding estimates, we introduce the sets

$$
\Omega_{j}(n)=\Omega_{j}^{-}(n) \cup \Omega_{j}^{+}(n), \quad j \in\{1,2,3,4\},
$$

where

$$
\Omega_{j}^{-}(n)=\left(-\frac{n}{2}+\frac{2 j}{\alpha} \log n,-\frac{2 j}{\alpha} \log n\right) \times\left(-\frac{n}{2}+\frac{2 j}{\alpha} \log n, \frac{n}{2}-\frac{2 j}{\alpha} \log n\right),
$$

and $\Omega_{j}^{+}(n)=-\Omega_{j}^{-}(n)$ is the mirror-image of $\Omega_{j}^{-}(n)$ with respect to the $y$-axis; the parameter $\alpha>0$ will be chosen as in (15) below. Note that, for $\alpha>0$ fixed, the sets $\Omega_{1}(n), \ldots, \Omega_{4}(n)$ are non-empty for $n$ large. We have the trivial inclusions $\Omega_{j+1}(n) \subset \Omega_{j}(n)$ for $j=1,2,3$.

We will use the following exponential decay estimate for the resolvent of the operators $H_{k}$. There are constants $C \geq 0, \alpha>0$ such that for any $E \in\left[a^{\prime}, b^{\prime}\right]$ and (measurable) sets $K_{1}, K_{2} \subset \mathbb{R}^{2}$ we have (cf., e.g., Proposition 2.4 in [1])

$$
\left\|\chi_{K_{1}} \partial_{j}^{p}\left(H_{k}-E\right)^{-1} \chi_{K_{2}}\right\| \leq C \mathrm{e}^{-\alpha \operatorname{dist}\left(K_{1}, K_{2}\right)}, \quad j, p \in\{0,1\}, k \in\{1,2\} ;
$$

here $\partial_{1}=\partial_{x}, \partial_{2}=\partial_{y}$. We also choose cut-off functions $\varphi_{n}, \psi_{n} \in C_{c}^{\infty}\left(\mathbb{R}^{2} ; \mathbb{R}\right)$ satisfying

$$
\operatorname{supp} \varphi_{n} \subset \Omega_{1}(n), \quad \varphi_{n} \uparrow \Omega_{2}(n)=1, \quad \operatorname{supp} \psi_{n} \subset \Omega_{3}(n), \quad \psi_{n} \uparrow \Omega_{4}(n)=1,
$$

and $\left|\nabla \varphi_{n}\right|,\left|\nabla \psi_{n}\right|,\left|\partial_{i j} \varphi_{n}\right|,\left|\partial_{i j} \psi_{n}\right| \leq c(\log n)^{-1}$ with some constant $c \geq 0$; here $\varphi_{n}=\varphi_{n, \ell}+\varphi_{n, r}$ with $\varphi_{n, \ell}$ and $\varphi_{n, r}$ being supported in $\Omega_{1}^{-}(n)$ and $\Omega_{1}^{+}(n)$, respectively. By a well-known argument we can now derive the desired localization property: by the Leibniz rule, we have for $i=1, \ldots, N(n)$

$$
\left(H_{1}-E_{i}\right)\left(\varphi_{n, \ell} u_{i, n}\right)=\left(H^{(n)}-E_{i}\right)\left(\varphi_{n, \ell} u_{i, n}\right)=-2 \nabla \varphi_{n, \ell} \cdot \nabla u_{i, n}-\Delta \varphi_{n, \ell} u_{i, n}
$$

so that

$$
\chi_{\Omega_{3}^{-}(n)} u_{i, n}=-\chi_{\Omega_{3}^{-}(n)}\left(H_{1}-E_{i}\right)^{-1} \chi_{\mathrm{supp} \nabla \varphi_{n, \ell}}\left[2 \nabla \varphi_{n, \ell} \cdot \nabla u_{i, n}+\Delta \varphi_{n, \ell} u_{i, n}\right] .
$$


Using that $\operatorname{dist}\left(\Omega_{3}(n), \operatorname{supp} \nabla \varphi_{n}\right) \geq 2 \alpha^{-1} \log n$ and $\left|\nabla \varphi_{n}\right|,\left|\Delta \varphi_{n}\right| \leq c(\log n)^{-1}$, the estimate (15) implies that

$$
\left\|\chi_{\Omega_{3}(n)} u_{i, n}\right\|, \quad\left\|\chi_{\Omega_{3}(n)} \nabla u_{i, n}\right\| \leq C\left(n^{2} \log n\right)^{-1}, \quad i=1, \ldots, N(n) .
$$

We now define $v_{i, n}=\left(1-\psi_{n}\right) u_{i, n}$ and let $\mathcal{M}_{n}=\operatorname{span}\left\{v_{i, n}: i=1, \ldots, N(n)\right\}$. We claim that

$$
\operatorname{dim} \mathcal{M}_{n}=N(n), \quad n \geq n_{0},
$$

for some $n_{0} \in \mathbb{N}$. Let $H_{Q_{n} \backslash \Omega_{4}(n)}$ be the operator $-\Delta+W$ on $Q_{n} \backslash \Omega_{4}(n)$ with Dirichlet boundary conditions. The functions $v_{i, n}=\left(1-\psi_{n}\right) u_{i, n}$ are approximate eigenfunctions of $H_{Q_{n} \backslash \Omega_{4}(n)}$ : in fact, using (16), one easily checks that

$$
\left\|\left(H_{Q_{n} \backslash \Omega_{4}(n)}-E_{i, n}\right) v_{i, n}\right\| \leq C\left(n^{2} \log ^{2} n\right)^{-1}
$$

and

$$
\left\|v_{i, n}-u_{i, n}\right\| \leq C\left(n^{2} \log n\right)^{-1},
$$

for $i=1, \ldots, N(n)$. Now (14) and (18) imply $\sum_{i=1}^{N(n)}\left\|u_{i, n}-v_{i, n}\right\|^{2}<1$ for $n$ large and we obtain (17).

(3) We next show that there is $n_{1} \geq n_{0} \in \mathbb{N}$ such that

$$
\left\langle H_{Q_{n} \backslash \Omega_{4}(n)} w, w\right\rangle<b\|w\|^{2}, \quad w \in \mathcal{M}_{n}, n \geq n_{1} .
$$

For a proof, consider an arbitrary $w=\sum_{i=1}^{N(n)} \gamma_{i} v_{i, n} \in \mathcal{M}_{n}$ with $\|w\|=1$. Here we first observe that the coefficients $\gamma_{i}$ satisfy a bound $\left|\gamma_{i}\right| \leq 2$, for $n$ large, since (writing $\gamma^{2}=\sum_{i}\left|\gamma_{i}\right|^{2}$ and $\eta_{n}^{2}=\sum_{i}\left\|v_{i, n}-u_{i, n}\right\|^{2}$ )

$$
1=\|w\| \geq\left\|\sum_{i=1}^{N(n)} \gamma_{i} u_{i, n}\right\|-\sum_{i=1}^{N(n)}\left|\gamma_{i}\right| \cdot\left\|v_{i, n}-u_{i, n}\right\| \geq \gamma\left(1-\eta_{n}\right),
$$

where $\eta_{n} \rightarrow 0$ as $n \rightarrow \infty$ by (18). Using (18) and the fact that $\nabla \psi_{n}$ and $\Delta \psi_{n}$ have support in $\Omega_{3}(n) \backslash \Omega_{4}(n)$, it follows that for $n$ large

$$
\|w\|^{2}=\sum_{i=1}^{N(n)}\left|\gamma_{i}\right|^{2}+r, \quad\left\langle H_{Q_{n} \backslash \Omega_{4}(n)} w, w\right\rangle=\sum_{i=1}^{N(n)} E_{i}\left|\gamma_{i}\right|^{2}+r^{\prime},
$$

where $r, r^{\prime} \leq C(\log n)^{-2}$, so that

$$
\left\langle H_{Q_{n} \backslash \Omega_{4}(n)} w, w\right\rangle \leq b^{\prime}\|w\|^{2}+r^{\prime \prime},
$$

with $r^{\prime \prime} \leq C(\log n)^{-2}$, for $n$ large, and we obtain (19).

(4) We conclude from (19) that $\mathcal{M}_{n} \subset P_{(-\infty, b)}\left(H_{Q_{n} \backslash \Omega_{4}(n)}\right)$ and then (17) implies that $\operatorname{dim} P_{(-\infty, b)}\left(H_{Q_{n} \backslash \Omega_{4}(n)}\right) \geq \operatorname{dim} \mathcal{M}_{n}=N(n)$. On the other hand, min-max arguments yield an upper bound for $\operatorname{dim} P_{(-\infty, b)}\left(H_{Q_{n} \backslash \Omega_{4}(n)}\right)$ of the form $c n \log n$, and we are done.

It seems to be possible, using more powerful methods, to remove the logarithmic factor in (11) and (13), see [4]. 


\section{Muffin tin potentials}

In this section, we present a class of examples where one can arrive at rather precise statements that illustrate some of the phenomena described before. Note that the results given below are derived directly, without recourse to Section 3. We will look at three types of muffin tin potentials and discuss the effect of the "filling up" of the gaps at small angles of rotation. We begin with muffin tins with walls of infinite height, then approximate by muffin tin potentials of height $n$, for $n \in \mathbb{N}$ large. By another approximation step, one may obtain examples with Lipschitz continuous potentials. These examples show, among other things, that Schrödinger operators of the form $R_{\vartheta}$ may in fact have spectral gaps for some $\vartheta>0$.

We consider the lattice $\mathbb{Z}^{2} \subset \mathbb{R}^{2}$ where we first introduce the Laplacian of a periodic muffin tin with infinitely high walls separating the wells: for $0<r<$ $1 / 2$, we let $D_{r}=B_{r}(1 / 2,1 / 2)$ denote the disc of radius $r$ centered at the point $(1 / 2,1 / 2) \in \mathbb{R}^{2}$, and generate from $D_{r}$ the periodic sets

$$
\Omega_{r}=\bigcup_{(i, j) \in \mathbb{Z}^{2}}\left(D_{r}+(i, j)\right), \quad 0<r<1 / 2 .
$$

The Dirichlet Laplacian $H_{r}$ of $\Omega_{r}$ is the direct sum of a countable number of copies of the Dirichlet Laplacian on $D_{r}$; therefore, the spectrum of $H_{r}$ consists in a sequence of positive eigenvalues $\left(\mu_{k}(r)\right)_{k \in \mathbb{N}}$ with $\mu_{k}(r) \rightarrow \infty$ as $k \rightarrow \infty$; we may assume that $\mu_{k}(r)<\mu_{k+1}(r)$ for all $k \in \mathbb{N}$. The eigenvalues $\mu_{k}=\mu_{k}(r)$ of $H_{r}$ have infinite multiplicity. The $\mu_{k}$ correspond to the bands of a periodic problem: in fact, defining $V_{r}: \mathbb{R}^{2} \rightarrow \mathbb{R}$ by

$$
V_{r}(x, y)= \begin{cases}0 & (x, y) \in \Omega_{r}, \\ 1 & (x, y) \notin \Omega_{r},\end{cases}
$$

the periodic Schrödinger operators $H_{r, n}=H_{0}+n V_{r}$ have purely a.c. spectrum with a band/gap structure. Furthermore, norm resolvent convergence $H_{r, n} \rightarrow H_{r}$, obtained as in [10] implies that the bands of $H_{r, n}$ converge to the eigenvalues $\mu_{k}$ of $H_{r}$. In the sequel, denote by $(a, b)$ one of the gaps $\left(\mu_{k}, \mu_{k+1}\right)$. We next look at the rotation problem where we define

$$
\Omega_{r, \vartheta}=\left(\Omega_{r} \cap\{x \geq 0\}\right) \cup\left(\left(M_{\vartheta} \Omega_{r}\right) \cap\{x<0\}\right) ;
$$

we also let $H_{r, \vartheta}$ denote the Dirichlet Laplacian on $\Omega_{r, \vartheta}$, for $0<r<1 / 2$ and $0 \leq \vartheta \leq \pi / 4$.

The set $\left(M_{\vartheta} \Omega_{r}\right) \cap\{x<0\}$ comes with two types of connected components: most (or, in some cases, all) components are discs, but typically there are also discs in $M_{\vartheta} \Omega_{r}$ with center at a distance less than $r$ from the $y$-axis; those appear in $\left(M_{\vartheta} \Omega_{r}\right) \cap\{x<0\}$ in a truncated form. It is then clear that $H_{r, \vartheta}$ has pure point spectrum. 
Let us comment on some special cases before we proceed: for $\tan \vartheta$ rational, these truncated discs form a periodic pattern; furthermore, we will find a half-disc in $\left(M_{\vartheta} \Omega_{r}\right) \cap\{x<0\}$ if and only if there is a disc in $M_{\vartheta} \Omega_{r}$ with center on the $y$-axis which happens if and only if $\tan \vartheta=1 /(2 k+1)$ for some $k \in \mathbb{N}$. It follows that for any $\tan \vartheta \in \mathbb{Q}$ with $\tan \vartheta \notin\{1 /(2 k+1): k \in \mathbb{N}\}$ there is some $r_{0}>0$ such that $n o$ component of $M_{\vartheta} \Omega_{r}$ meets the $y$-axis, for $0<r<r_{0}$; in other words, in this case all components of $\Omega_{r, \vartheta}$ are discs.

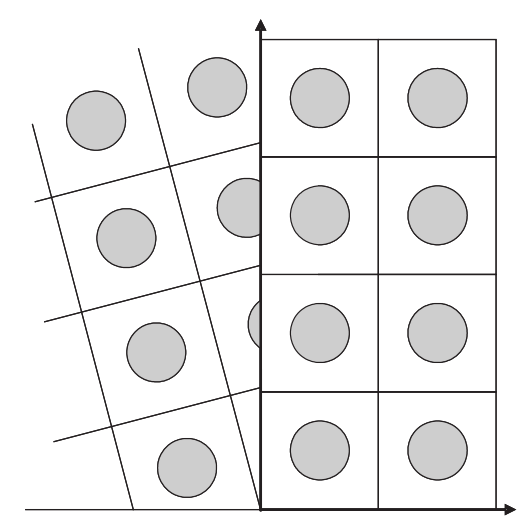

Figure 2. The domain $\Omega_{1 / 4, \pi / 8}$ (shaded).

We now return to the general situation. Moving up the $y$-axis from the origin, we denote the discs in $M_{\vartheta} \Omega_{r}$ that intersect the $y$-axis by $D_{j ; r, \vartheta}, j \in J_{\vartheta}$, with centers $\left(\xi_{j ; \vartheta}, \eta_{j ; \vartheta}\right)$; here $J_{\vartheta}=\emptyset$ or $J_{\vartheta}=\mathbb{N}$ according to the cases discussed above. Without restriction, we may assume that the $\eta_{j ; \vartheta}$ are monotonically increasing. Let

$$
C_{j ; r, \vartheta}=D_{j ; r, \vartheta} \cap\{x<0\}, \quad j \in J_{\vartheta} .
$$

Clearly, the Dirichlet eigenvalues of the components $C_{j ; r, \vartheta}$ are the surface states in this model. Since the eigenvalues of the $C_{j ; r, \vartheta}$ only depend on $r$ and $\xi_{j ; \vartheta}$ (but not on $\eta_{j ; \vartheta}$ ), it is enough to study the eigenvalues of the following sample domains. For $r>0$ and $-r<\xi \leq r$, we denote

$$
C_{r, \xi}=\left\{(x, y) \in D_{r}: x<1 / 2+\xi\right\} .
$$

The Dirichlet Laplacian of $C_{r, \xi}$ has eigenvalues $\lambda_{k}(r, \xi), k \in \mathbb{N}$, which depend continuously on $\xi$, for $k \in \mathbb{N}$ and $r>0$ fixed. Furthermore, $\lambda_{k}(r, \xi) \rightarrow \infty$ as $\xi \rightarrow-r$ and $\lambda_{k}(r, \xi) \rightarrow \mu_{k}(r)$, as $\xi \rightarrow r$. Therefore, for each $k \in \mathbb{N}$, the eigenvalues $\lambda_{k}(r, \xi)$ range over the interval $\left[\mu_{k}(r), \infty\right)$.

We now combine the above properties of $\lambda_{k}(r, \xi)$ with information on the distribution of the coordinates $\xi_{j ; \vartheta}$. It is easy to see that, as $0 \neq \vartheta \rightarrow 0$, the $\xi_{j ; \vartheta}$ partition the interval $(-r, r)$ into subintervals of smaller and smaller length. Therefore, for 
$\varepsilon>0$ given, any interval $(\alpha, \beta) \subset(a, b)$ of length $\geq \varepsilon$ will contain an eigenvalue of $H_{r, \vartheta}$ for all sufficiently small $\vartheta>0$.

We expect stronger properties for angles $\vartheta$ for which the set $\left\{\xi_{j ; \vartheta}: j \in \mathbb{N}\right\}$ is dense in $(-r, r)$. As in Section 3, ergodic theory gives us a set $\Theta \subset(0, \pi / 2)$ of full measure such that for each $\vartheta \in \Theta$ the set $\left\{(m \tan \vartheta)_{\sim}: m \in \mathbb{N}\right\}$ is dense in $(0,1)$, which implies the desired property for the $\xi_{j ; \vartheta}$. It follows that, for any $\vartheta \in \Theta$, the eigenvalues of $H_{r, \vartheta}$ will be dense in $\left[\mu_{1}(r), \infty\right)$.

Finally, for $\tan \vartheta$ rational the $\xi_{j ; \vartheta}$ form a periodic set and then $H_{r, \vartheta}$ will only have a finite number of eigenvalues (each of infinite multiplicity) in the gap $(a, b)$. We thus have the following result.

Proposition 5.1. Let $0<r<1 / 2$ be fixed.

(a) Each $\mu_{k}(r), k=1,2, \ldots$, is an eigenvalue of infinite multiplicity of $H_{r, \vartheta}$, for all $0 \leq \vartheta \leq \pi / 4$. The spectrum of $H_{r, \vartheta}$ is pure point, for all $0 \leq \vartheta \leq \pi / 4$.

(b) For any $\varepsilon>0$ there is a $\vartheta_{\varepsilon}=\vartheta_{\varepsilon}(r)>0$ such that any interval $(\alpha, \beta) \subset(a, b)$ with $\beta-\alpha \geq \varepsilon$ contains an eigenvalue of $H_{r, \vartheta}$ for any $0<\vartheta<\vartheta_{\varepsilon}$.

(c) There exists a set $\Theta \subset(0, \pi / 2)$ offull measure such that $\sigma\left(H_{r, \vartheta}\right)=\left[\mu_{1}(r), \infty\right)$. The eigenvalues different from the $\mu_{k}(r)$ are of finite multiplicity.

Remark 5.2. Let $\Lambda=\{\vartheta \in(0, \pi / 2): \tan \vartheta \in \mathbb{Q}\}$ denote the set of angles where $\tan \vartheta$ is rational; clearly, $\Theta \cap \Lambda=\varnothing$. It is easy to see that $H_{r, \vartheta}$, for $\vartheta \in \Lambda$, has at most a finite number of eigenvalues in $(a, b)$, each of them of infinite multiplicity. Hence we see a drastic change in the spectrum for $\vartheta \in \Lambda$ as compared with $\vartheta \in \Theta$. Furthermore, if $\vartheta \in \Lambda$ with $\tan \vartheta \notin\{1 /(2 k+1): k \in \mathbb{N}\}$, then there is some $r_{\vartheta}>0$ such that $\sigma\left(H_{r, \vartheta}\right)=\sigma\left(H_{r}\right)$ for all $0<r<r_{\vartheta}$.

We next turn to muffin tin potentials of finite height. Here we define the potential $V_{r, \vartheta}$ to be zero on $\Omega_{r, \vartheta}$ and $V_{r, \vartheta}=1$ on the complement of $\Omega_{r, \vartheta}$, where $0<r<1 / 2$ and $0 \leq \vartheta \leq \pi / 4$; we also let $H_{r, n, \vartheta}=H_{0}+n V_{r, \vartheta}$. The periodic operators $H_{r, n, 0}$ have purely absolutely continuous spectrum.

We first show that, for $r, \vartheta$ fixed, the operators $H_{r, n, \vartheta}$ converge to $H_{r, \vartheta}$ in the sense of norm resolvent convergence. This can be seen as follows. In view of Theorem 7.1, we introduce an additional Dirichlet boundary condition on a (closed) set $S=S_{\vartheta} \subset \mathbb{R}^{2} \backslash \Omega_{r, \vartheta}$, which we now define. Let $\Gamma \subset \mathbb{R}^{2}$ denote the grid $\left\{(x, y) \in \mathbb{R}^{2}: x \in \mathbb{Z}\right.$ or $\left.y \in \mathbb{Z}\right\}$, and let $\Gamma_{\vartheta}=M_{\vartheta} \Gamma$ denote the rotated grid. For $\rho=(1 / 2-r) / 2$, we let $S$ consist of $\Gamma \cap\{(x, y): x>\rho\}, \Gamma_{\vartheta} \cap\{x<\rho\}$ plus the vertical line $\{x=\rho\}$. Note that $S$ has distance $\rho>0$ from $\Omega_{r, \vartheta}$. Let $H_{r, n, \vartheta ; S}$ denote the operator $-\Delta+n V_{r, \vartheta}$ with Dirichlet boundary condition on $S$. We now have

$$
\begin{aligned}
\left\|\left(H_{r, \vartheta}+1\right)^{-1} \oplus 0-\left(H_{r, n, \vartheta}+1\right)^{-1}\right\| \leq & \left\|\left(H_{r, \vartheta}+1\right)^{-1} \oplus 0-\left(H_{r, n, \vartheta ; S}+1\right)^{-1}\right\| \\
& +\left\|\left(H_{r, n, \vartheta ; S}+1\right)^{-1}-\left(H_{r, n, \vartheta}+1\right)^{-1}\right\|,
\end{aligned}
$$

where 0 denotes the zero operator on $L_{2}\left(\mathbb{R}^{2} \backslash \Omega_{r, \vartheta}\right)$. By Theorem 7.1, applied with $U=\mathbb{R}^{2} \backslash \bar{\Omega}_{r, \vartheta}$, we can find $n_{0} \in \mathbb{N}$ such that the second term on the RHS is smaller 
than any given $\varepsilon>0$, for $n \geq n_{0}$. The first term on the RHS is a direct sum of operators living on the components of $\mathbb{R}^{2} \backslash S_{\vartheta}$. By routine arguments, we have norm resolvent convergence on each of the components, as $n \rightarrow \infty$, and all we have to do is to convince ourselves that this convergence holds uniformly on all components. The components of $\mathbb{R}^{2} \backslash S_{\vartheta}$ fall into 4 classes: there are unrotated and rotated squares, there are rectangles in the right half-plane of the form $(\rho, 1) \times(\ell, \ell+1)$ for $\ell \in \mathbb{Z}$, and there are polygons (triangles, quadrangles, and pentagons) in the set $\{x<\rho\}$ that are bounded to the right by the line $x=\rho$. We have no problem with uniform convergence for the first three classes and Lemma 7.3 takes care of the fourth class. It is easy to see that the norm resolvent convergence $H_{r, n, \vartheta} \rightarrow H_{r, \vartheta}$ as $n \rightarrow \infty$ is uniform in $\vartheta \in[0, \pi / 4]$. We then obtain from Proposition 5.1(b) and Remark 5.2 the following results.

Proposition 5.3. (a) For $\tan \vartheta \in \mathbb{Q}$ the spectrum of $H_{r, n, \vartheta}$ has gaps inside the interval $(a, b)$ for $n$ large. More precisely, if $H_{r, \vartheta}$ has a gap $\left(a^{\prime}, b^{\prime}\right) \subset(a, b)$, then, for $\varepsilon>0$ given, the interval $\left(a^{\prime}+\varepsilon, b^{\prime}-\varepsilon\right)$ will be free of spectrum of $H_{r, n, \vartheta}$ for $n$ large.

(b) For any $\varepsilon>0$ there are $\vartheta_{0}>0$ and $n_{0}>0$ such that any interval $(c-\varepsilon$, $c+\varepsilon) \subset(a, b)$ contains spectrum of $H_{r, n, \vartheta}$ for all $0<\vartheta<\vartheta_{0}$ and $n \geq n_{0}$.

By similar arguments, we can approximate $V_{r, \vartheta}$ by Lipschitz-continuous muffin tin potentials that converge monotonically (from below) to $V_{r, \vartheta}$ in such a way that norm resolvent convergence holds for the associated Schrödinger operators (again uniformly in $\vartheta \in[0, \pi / 4])$. The spectral properties obtained are analogous to the ones stated in Proposition 5.3. Note, however, that the statement corresponding to part (b) in Proposition 5.3 is weaker than the result of our main Theorem 1.1.

A brief study of translational dislocation problems for muffin tin potentials can be found in [12].

\section{Some extensions and remarks}

(1) A simple variant of the rotation problem consists in rotations in the left and the right half planes through angles $\vartheta / 2$ and $-\vartheta / 2$, respectively, i.e., we study

$$
\tilde{V}_{\vartheta}(x, y)= \begin{cases}\left(V \circ M_{-\vartheta / 2}\right)(x, y) & x \geq 0, \\ \left(V \circ M_{\vartheta / 2}\right)(x, y) & x<0 ;\end{cases}
$$

this potential might be rather close to the physical situation shown in Figure 1. Here we consider the accompanying translational dislocation potentials

$$
\tilde{W}_{t}(x, y)= \begin{cases}V(x-t / 2, y) & x \geq 0, \\ V(x+t / 2, y) & x<0 .\end{cases}
$$


We may then obtain results as in Theorem 1.1 without the use of Birkhoff's theorem: here we only need to take care of the second condition in (9) since the horizontal alignment between the left- and right-hand part of $V_{\vartheta}$ on the $y$-axis is guaranteed by the definition of $\widetilde{V}_{\vartheta}$.

(2) We have shown that the spectral gaps of $H$ fill with spectrum of $R_{\vartheta}$ as $\vartheta \rightarrow 0$ in the sense that any interval of length $\varepsilon>0$ inside a gap of $H$ will contain spectrum of $R_{\vartheta}$ for sufficiently small angles. In general, we do not know whether the spectrum of $R_{\vartheta}$ in the gaps of $H_{0}$ is pure point, absolutely continuous or singular continuous. However, there are some special angles where we can exclude singular continuous spectrum: if we assume that $\cos \vartheta$ is a rational number, $\cos \vartheta=q / p$ with $p, q \in \mathbb{N}$, and $p$ and $q$ belong to a Pythagorean triple $\left(p^{2}-q^{2}=r^{2}\right.$ for some $\left.r \in \mathbb{N}\right)$, then $V_{\vartheta}$ has period $p$ in $y$-direction. In this case, a result in [6] implies that $\sigma\left(H_{\vartheta}\right)$ has no singular continuous part.

(3) It is natural to ask about higher dimensions. Suppose we are given a potential $V: \mathbb{R}^{3} \rightarrow \mathbb{R}$, periodic with respect to the lattice $\mathbb{Z}^{3}$. We may then simply consider rotations of the $(x, y)$-plane by an angle $\vartheta$, i.e. we let $V_{\vartheta}(x, y, z)=V(x, y, z)$ in $\{(x, y, z): x \geq 0\}$ and $V_{\vartheta}(x, y, z)=V\left(M_{-\vartheta}(x, y), z\right)$ in $\{(x, y, z): x<0\}$, in which case our methods should apply. However, in $\mathbb{R}^{3}$ there are many other rotations for which our methods may or may not work.

(4) Of course, taking the limit $\vartheta \rightarrow 0$ is a mathematical idealization. In real crystals or alloys the lattice and its rotated version have to match up according to certain rules. This is usually only possible for a small number of angles. Related questions in higher dimensions are studied under the name of coincidence site lattices (CSL); cf. [3] and [23].

\section{Appendix}

In this brief appendix we prove a - rather general - result on decoupling by Dirichlet boundary conditions placed on a set inside a high barrier. The method of proof is fairly standard but we have been unable to find a suitable reference which would have covered our situation. Let $d \in \mathbb{N}$. For some open set $U \subset \mathbb{R}^{d}$ and a closed set $S \subset U$ of measure zero, we consider for $n \in \mathbb{N}$ the Schrödinger operators $H_{n}=-\Delta+n \chi_{U}$, acting in $L_{2}\left(\mathbb{R}^{d}\right)$, and $H_{n, S}=-\Delta+n \chi_{U}$ in $L_{2}\left(\mathbb{R}^{d} \backslash S\right)=L_{2}\left(\mathbb{R}^{d}\right)$, where $H_{n, S}$ is assumed to obey Dirichlet boundary conditions on the set $S$. In other words, the associated quadratic forms $h_{n}$ and $h_{n, S}$ have $C_{c}^{\infty}\left(\mathbb{R}^{d}\right)$ and $C_{c}^{\infty}\left(\mathbb{R}^{d} \backslash S\right)$ as form cores. We then show that the resolvent difference $\left(H_{n}+1\right)^{-1}-\left(H_{n, S}+1\right)^{-1}$ goes to zero in norm, as $n \rightarrow \infty$, provided the set $S$ has a positive distance to the boundary of $U$; note that the set $S$ need not be bounded.

Theorem 7.1. Let $H_{n}$ and $H_{n, S}$ as above and suppose that $\operatorname{dist}(S, \partial U)>0$. Then

$$
\left\|\left(H_{n}+1\right)^{-1}-\left(H_{n, S}+1\right)^{-1}\right\| \rightarrow 0, \quad n \rightarrow \infty .
$$


Proof. For $f \in L_{2}\left(\mathbb{R}^{d}\right)$ with $\|f\| \leq 1$, let $u_{n}=\left(H_{n}+1\right)^{-1} f$ and let $v_{n}=$ $\left(H_{n, S}+1\right)^{-1} f$. We then have the trivial estimates $\left\|u_{n}\right\| \leq 1$ and

$$
\left\|\nabla u_{n}\right\|^{2}+n \int_{U}\left|u_{n}\right|^{2} d x=h_{n}\left[u_{n}\right] \leq\left\langle\left(H_{n}+1\right) u_{n}, u_{n}\right\rangle=\left\langle f, u_{n}\right\rangle \leq 1,
$$

so that $\left\|\nabla u_{n}\right\| \leq 1$ and $\int_{U}\left|u_{n}\right|^{2} d x \leq 1 / n$, for all $n \in \mathbb{N}$; analogous estimates hold for $v_{n}$. We let $\mathcal{F}$ denote the set of all functions $\varphi \in C^{\infty}\left(\mathbb{R}^{d}\right)$ that satisfy supp $\varphi \subset U$, $\operatorname{dist}(\operatorname{supp} \varphi, \partial U)>0,0 \leq \varphi \leq 1$, and $\nabla \varphi, \Delta \varphi$ bounded. For any $\varphi \in \mathscr{F}$, we then have $\varphi u_{n} \in D\left(H_{n}\right)$ and $\left(H_{n}+1\right)\left(\varphi u_{n}\right)=\varphi f-2 \nabla u_{n} \nabla \varphi-u_{n} \Delta \varphi$. We immediately see from the above estimates that there is a constant $c_{\varphi} \geq 0$ such that

$$
\left\|H_{n}\left(\varphi u_{n}\right)\right\| \leq c_{\varphi} .
$$

We now derive a crucial estimate for $\left\|\nabla\left(\varphi u_{n}\right)\right\|$. Since $\left\|\nabla\left(\varphi u_{n}\right)\right\|^{2} \leq h_{n}\left[\varphi u_{n}\right]=$ $\left\langle H_{n}\left(\varphi u_{n}\right), \varphi u_{n}\right\rangle$, for any $\varepsilon>0$ there are constants $C_{\varepsilon}, C_{\varepsilon}^{\prime} \geq 0$ such that

$$
\left\|\nabla\left(\varphi u_{n}\right)\right\|^{2} \leq \varepsilon\left\|H_{n}\left(\varphi u_{n}\right)\right\|^{2}+C_{\varepsilon}\left\|\varphi u_{n}\right\|^{2} \leq \varepsilon c_{\varphi}^{2}+C_{\varepsilon}^{\prime} / n .
$$

Analogous estimates hold for $H_{n, S}$ and $v_{n}$.

We let $\rho=\operatorname{dist}(S, \partial U), U_{\rho}=\{x \in U: \operatorname{dist}(x, S)<\rho / 2\}$ and fix some function $\psi \in \mathcal{F}$ with $\psi=1$ on $U_{\rho}$. Let $\eta=1-\psi$ and choose another function $\varphi_{\psi} \in \mathcal{F}$ satisfying $\varphi_{\psi}=1$ on the support of $\nabla \psi$. Writing $w_{n}=u_{n}-v_{n}=\left(H_{n}+1\right)^{-1} f-$ $\left(H_{n, S}+1\right)^{-1} f$ it is then clear that $\psi w_{n} \rightarrow 0$, as $n \rightarrow \infty$, uniformly for all $f$ with $\|f\| \leq 1$, and it remains to consider $\eta w_{n}$. Since $\eta$ vanishes on $U_{\rho}$, we have $\eta w_{n} \in D\left(H_{n}\right)$ and

$$
\left(H_{n}+1\right)\left(\eta w_{n}\right)=-2 \nabla \eta \cdot \nabla w_{n}-(\Delta \eta) w_{n}=: z_{n},
$$

so that, in particular,

$$
\eta w_{n}=\left(H_{n}+1\right)^{-1} z_{n}, \quad\left\|\eta w_{n}\right\| \leq\left\|z_{n}\right\| .
$$

We finally show that $z_{n} \rightarrow 0$, uniformly for $\|f\| \leq 1$. Here we know already that $(\Delta \eta) w_{n} \rightarrow 0$ since $\Delta \eta$ is supported inside $U$. Applying the estimate (21) we find that

$$
\left\|\nabla \eta \cdot \nabla w_{n}\right\| \leq C_{\eta}\left\|\nabla\left(\varphi_{\psi} w_{n}\right)\right\| \leq \varepsilon c_{\psi}+C_{\varepsilon, \psi}^{\prime} / n,
$$

with $c_{\psi}$ as in (20) and (21). For $\delta>0$ given, we can find $\varepsilon>0$ s.th. $\varepsilon c_{\psi}<\delta / 2$ and then $n_{0} \in \mathbb{N}$ such that $C_{\varepsilon, \psi}^{\prime} / n<\delta / 2$ for all $n \geq n_{0}$, and we are done.

Remarks 7.2. (a) The assumption that $S$ has measure zero is made chiefly for simplicity of notation.

(b) The same result holds for Schrödinger operators $-\Delta+n V$ where $V: \mathbb{R}^{d} \rightarrow \mathbb{R}$ is a non-negative, bounded potential satisfying $V \geq \chi_{U}$. Our method of proof yields a bound on the norm of the resolvent difference which is independent of $V$.

(c) It is well-known that $H_{n}$ and $H_{n, S}$ converge to a suitably defined Dirichlet Laplacians in the sense of strong resolvent convergence, cf. [13]. For periodic $U$ the convergence would even be in the norm resolvent sense, cf. [10]. 
We finally discuss uniform norm resolvent convergence for Schrödinger operators on domains of the type encountered at the end of Section 5. Here we will use the well-known fact that a monotonic sequence $\left(A_{n}\right)_{n \in \mathbb{N}}$ of compact operators which converges strongly to a (compact) operator $A$ converges in norm, i.e. $\left\|A_{n}-A\right\| \rightarrow 0$.

For simplicity of notation, we consider an equivalent geometric situation with a family of domains in the first quadrant given as follows.

We fix $0<\vartheta<\pi / 4$ and $0<r, d<1 / 2$. Then, for $s \in \mathbb{R}$, we consider two parallel lines $\ell_{1, s}, \ell_{2, s}$ defined, respectively, by the equations $y=(x-s) \cot \vartheta$ and $y=(x-s) \cot \vartheta+d / \sin \vartheta$ so that $\ell_{1, s}$ and $\ell_{2, s}$ have distance $d$. We let

$$
G_{s}=[0,1]^{2} \cap\left\{(x, y) ; y>\ell_{1, s}(x)\right\}, \quad D_{r, s}=B_{r}\left(\frac{1}{2}, \frac{1}{2}\right) \cap\left\{(x, y) ; y>\ell_{2, s}(x)\right\} ;
$$

cf. Figure 3. Note that $D_{r, s}$ (if it is non-empty) has distance $\min \{d, 1-r\}$ to the boundary of $G_{s}$.

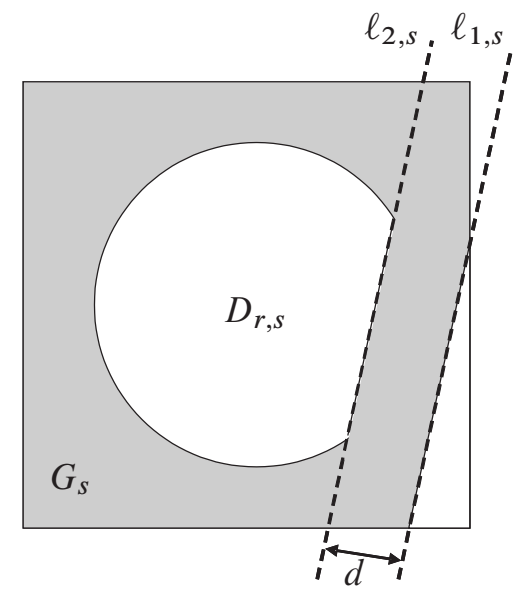

Figure 3. A typical domain $D_{r, s}$ (shaded).

We next define quadratic forms on the domains $G_{s}$ : for $n \in \mathbb{N}$, we let $V_{r, s, n}=0$ on $D_{r, s}$ and $V_{r, s, n}=n$ on $G_{s} \backslash D_{r, s}$. As $s$ increases, the sets $G_{s}$ and $D_{r, s}$ both increase and we therefore see that the quadratic forms

$$
h_{r, s, n}[\varphi]=\|\nabla \varphi\|^{2}+\int_{G_{s}} V_{n}|\varphi|^{2} \mathrm{~d} x, \quad \varphi \in C_{c}^{\infty}\left(G_{s}\right),
$$

depend monotonically on $s$. It is easy to include the case $n=\infty$ by setting

$$
h_{r, s, \infty}[\varphi]=\|\nabla \varphi\|^{2}, \quad \varphi \in C_{c}^{\infty}\left(D_{r, s}\right) .
$$

The self-adjoint operators $H_{r, s, n}$ and $H_{r, s, \infty}$ associated with (the closure of) these quadratic forms have compact resolvent. We have the following result. 
Lemma 7.3. With the above definitions and assumptions, we have

$$
\sup _{s}\left\|H_{r, s, n}^{-1}-H_{r, s, \infty}^{-1}\right\| \rightarrow 0, \quad n \rightarrow \infty .
$$

Proof. Obviously, we may restrict our attention to parameters $s$ from a compact interval $J \subset \mathbb{R}$. Writing

$$
f_{n}(s)=\left\|H_{r, s, n}^{-1}-H_{r, s, \infty}^{-1}\right\|, \quad s \in J
$$

monotonicity and compactness imply that the functions $f_{n}$ are continuous with $f_{n}(s) \rightarrow 0$ monotonically as $n \rightarrow \infty$, for each fixed $s$. Now the desired result follows by Dini's theorem.

\section{References}

[1] S. Alama, M. Avellanda, P. A. Deift, and. R Hempel, On the existence of eigenvalues of a divergence form operator $A+\lambda B$ in a gap of $\sigma(A)$. Asymptotic Anal. 8 (1994), 311-344. MR 1278777 Zbl 0806.47042

[2] S. Alama, P. A. Deift, and R. Hempel, Eigenvalue branches of the Schrödinger operator $H-\lambda W$ in a gap of $\sigma(H)$. Commun. Math. Phys. 121 (1989), 291-321. MR 0985401 Zbl 0676.47032

[3] M. Baake, Solution of the coincidence problem in dimensions $d \leq 4$. In R. V. Moody (ed.), The mathematics of long-range aperiodic order. Proceedings of the NATO Advanced Study Institute, Waterloo, Ontario, Canada, August 21-September 1, 1995. Dordrecht, 1997, pp. 9-44. MR 1460018 Zbl 0883.20020

[4] H. Cornean, private communication, 2010.

[5] I. P. Cornfield, S. V. Fomin, and Y. G. Sinai, Ergodic theory, Springer Verlag, New York, 1982. MR 0832433 Zbl 0493.28007

[6] E. B. Davies, and B. Simon, Scattering theory for systems with different spatial asymptotics on the left and right. Commun. Math. Phys. 63 (1978), 277-301. MR 0513906 Zbl 0393.34015

[7] P. A. Deift and R. Hempel, On the existence of eigenvalues of the Schrödinger operator $H-\lambda W$ in a gap of $\sigma(H)$, Commun. Math. Phys. 103 (1986), 461-490. MR 0832922 Zbl 0594.34022

[8] M. S. P. Eastham, The spectral theory of periodic differential equations. Scottish Academic Press, Edinburgh and London, 1973. Zbl 0287.34016

[9] H. Englisch, W. Kirsch, M. Schröder, and B. Simon, Random Hamiltonians ergodic in all but one direction. Commun. Math. Phys. 128 (1990), 613-625. MR 1045887 Zbl 0691.60053

[10] R. Hempel and I. Herbst, Strong magnetic fields, Dirichlet boundaries, and spectral gaps. Commun. Math. Phys. 169 (1995), 237-259. MR 1329195 Zbl 0827.35031 
[11] R. Hempel, A. Hinz, and H. Kalf, On the essential spectrum of Schrödinger operators with spherically symmetric potentials. Math. Ann. 277 (1987), 197-208. MR 0886419 Zbl 0629.35028

[12] R. Hempel and M. Kohlmann, A variational approach to dislocation problems for periodic Schrödinger operators. J. Math. Anal. Appl. 381 (2011), 166-178. Zbl 05904460

[13] I. Herbst and Z. Zhao, Sobolev spaces, Kac-regularity, and the Feynman-Kac formula. In E. Çinlar, K. L. Chung, and R. K. Getoor (eds.), Seminar on stochastic processes, 1987. (7th seminar, Princeton University, New Jersey, March 26-28, 1987). Managing ed.: J. Clover. Birkäuser, Boston etc., 1988, pp. 171-191. MR 1046416 Zbl 0656.60089

[14] T. Kato, Perturbation theory for linear operators. Springer Verlag, Berlin etc., 1966. MR 0203473 Zbl 0148.12601

[15] Ch. Kittel, Introduction to Solid State Physics, John Wiley and Sons, London, 1953. Zbl 0052.45506

[16] E. Korotyaev, Lattice dislocations in a 1-dimensional model, Commun. Math. Phys. 213 (2000), 471-489. MR 1785464

[17] E. Korotyaev, Schrödinger operators with a junction of two 1-dimensional periodic potentials. Asymptotic Anal. 45 (2005), 73-97. MR 2181260 Zbl 0962.34064

[18] V. Kostrykin and R. Schrader, Regularity of the surface density of states. J. Funct. Anal. 187 (2001), 227-246. MR 1867350 Zbl 1005.82015

[19] P. Kuchment, Floquet Theory for Partial Differential Equations, Birkhäuser, Basel etc., 1993. MR $1232660 \mathrm{Zbl} 0789.35002$

[20] M. Reed and B. Simon, Methods of modern mathematical physics I: Functional analysis. Rev. and enl. ed. Academic Press, New York etc., 1980. MR 0529429 Zbl 0459.46001

[21] M. Reed and B. Simon, Methods of modern mathematical physics. IV: Analysis of operators. Academic Press, New York etc., 1978. MR 0493421 Zbl 0401.47001

[22] I. Veselic, Existence and regularity properties of the integrated density of states of random Schrödinger operators. Springer Verlag, Berlin, 2008. Zbl 1189.82004

[23] P. Zeiner, Multiple CSLs for the body centered cubic lattice. J. Physics, Conference series 30 (2006), 163-167.

Received October 11, 2010; revised December 21, 2010

Rainer Hempel, Institute for Computational Mathematics, Technische Universität

Braunschweig, Pockelsstraße 14, 38106 Braunschweig, Germany

E-mail: r.hempel@tu-bs.de

Martin Kohlmann, Institute for Applied Mathematics, Leibniz Universität Hannover,

Welfengarten 1, 30167 Hannover, Germany

E-mail: kohlmann@ifam.uni-hannover.de 